\title{
Evidence of Cxcr4 Gene, Pecam1 Gene, Icam1 Gene, from Cells Showing the Antigen, in Invertebrates
}

\author{
Michel Leclerc \\ 556 rue Isabelle Romée, 45640 Sandillon (France) \\ *Corresponding Author: Michel Leclerc, 556 rue Isabelle Romée, 45640 Sandillon (France)
}

\begin{abstract}
Many genes which are present in Human are also present in Echinodermata such as CXCR4 gene, PECAM1 gene and ICAMI gene:they are characteristics of genes showing the antigen.2 Echinodermata genomes were studied: Ophiocomina nigra (Ophuirids) one and Antedon bifida (Crinoïds) one.The transcriptome sequences in 5'-3' were described in this paper.

Keywords: Invertebrates ; Echinodermata ; CXCR4 gene, PECAM1 gene;ICAM1 gene.
\end{abstract}

\section{INTRODUCTION}

CXCR4 gene, PECAM1 gene and ICAM1 gene are a typical ones we met usually in Human. The CXCR4 gene provides instructions for making a receptor protein that spans the outer membrane of cells specifically white blood cell.The protein encoded by PECAM1 gene was found on platelets (Invertebrate platelets were recently discovered (Ref .1) and T lymphocytes which exist also in Invertebrates .As for ICAM1 gene, it encodes for a ICAM1 glycoprotein which is situated on monocytes (in human).

Since we discovered Invertebrate Primitive Antibody (Ref. 2-3) and invertebrrate lymphocytes in Echinodermata, we decide to look for genes and cells which are implicated in » showing the antigen ».

Ophuirid and Crinoïd genomes were studied.

\section{Materials ANd Methods}

Animals : Ophiocomina nigra (Ophuirid) Antedon bifida(Crinoïd) were obtained at the station «Of Biologie Marine of Roscoff » France.

Obtention of Ophuirid and Crinoïd Mrna: Digestive coeca were excised from their bodies and mRNA were obtained from Uptizol (Interchim) then quality controls were operated.

Sequencing : Sequencing was made on Illumina Next Seq 500 with paired-end: 2. 75 bp Transcriptome was assembled from RNA-Seq fastq files using Trinity v2.1.1 ( Ref.4) with default parameters. A BLAST database was created with the assembled transcripts using makeblastdb application from ncbi-blast+ (v2.2.31+). The sequences of transcripts of interest were then blasted against this database using blastn application from ncbi-blast+ ( Ref.5) with parameter word_size 7 .

\section{RESULTS}

Various Echinodermata transcriptomes of Antedon bifida and Ophiocomina nigra are summarized :

CXCR4 transcriptome in Antedon bifida (Crinoïd, Echinodermata)

The corresponding sequence in 5'-3' shows :

>TRINITY_DN12629_c0_g2_i1 (CXCR4)

5'AAAATGAAAAATGATACAACTTTTATTTTT TATATGATTCCTTCAGTAATGGTAGGTCTA

TATGAAAGTTGATTAGGTAATACATTCTTA TATACTGTTCTTAATTGCAACCAGTAATAT

TTTAAGTACAGACTACTACTCCACATATTT GTATATTGATACAGTATTCCAAAATTGGTG

TATTGCAGTGTACAGTACTTTGTATTAAGT TGTATGGGACTTATTTTTTAGCATTATATT

ACTGTAATACTTGACATGTTCCACTAGTTT GTCCCGGTCTTCTTCTGGGAGAAATGCACA

TTCAGCAGCACAAACTCTTAATCTTATCACA TCTTCATCCGATATTCCAAAATATTTCAG

AGCAATATCCTCTTCTGTCATTAATGTGTT CATAAAATGCAAACTGTCGTCAGTATTTAA

TGAAAAATTCAAACTATCTTTTGCAAATC TTATAGCTGGATGTTTATTCCAATCTGGACT

AACACTACCAAGAATCATACTAGAGGTTG GGCATACTTCCAAATGTACATTTGTCTTCTT

TACCAACTCATAAACTTTGTCATCATCAAGA CAATGATAACCGTGACCTATACGTTGAGC 
Evidence of Cxcr4 Gene, Pecam1 Gene, Icam1 Gene, from Cells Showing the Antigen, in Invertebrates

TTTAAACACCTCAATTGCTTCCTTTACATT GGCTGCTGGTCCGGACTCCCCTGCATGTAT

TGCTCTCTTTATGTTTAGTTTAATGGCTTC CTGAAATGCCTGGTGAAATTCATTTTGTAG

AGTTAGTGATTCGTCACCACCTATACCAATG CCTACAACACCATCATTTCGATACTTGTC

ACATAATTCTACAACCTCCTGGCACCACT CTGGCTTTCCTCTCATAAGACATATTATACT

CCTGGCTTGTACACCATACTTGTCTTGTCCT TCTTTTAAACCTTCATTCACAAGTTGTAC

AACTTGATCTGCTGTCATATTTTCATTTGA TAGAAGATGGGGGCAGTATGATGTTTCGAA

ATATGCAACCCCTTCCTTCAACTTATCTTC ACACAGATCTCTTGCAATTTCCTTTATTGC

TTCACTATCACCTCTTAGAATAGGCATATA GATTTCAAAACTCTTGATGAATTTTGTCAA

TGATCCACCACCCACTACATGTAGCTCTGA AGTGAAGTCTTTAAAGTTCTTTCCAGGCAG

TGTATCTAACATGCCTCGTCGTTTTGCAATG TTCCAAAGAGTTTCTGCCCGACAAGAACC

ATCAAGGTGGCAATGTAATTCAACCTAAAA TAAAAACCAATTCAACACATCCATAACATT

\section{AAAT3'}

The sequence of PECAM1 Transcriptome in 5'3 ' in Ophiocomina genome is following :

5'ATATATCATATATGATATAGTACCTTT GTTATATATCATAATACATATAAATGTGT ATTA

TGTTATCTATAATTATATAATTTCATATAT AAGATGTATAATATGTATCATATATTATAT

ATGTTATGTAATATATATAGTATATATAA GATGACACAGGATAAATATTATATACTA TGA

CATATAAAATATATGAGGTTATATGTTA CATATAAGGCATAGCACATAACATGTAA TATA

TATCATATATAATTTTTTTTTAGACAGAAT CTTGTCCTGTTGCACAGGGTGGGGTACAAT GGCGCCATCTTTGCTCACTGCAACTTCTGC CTCACGGGTCCAAGCGATTGTCCTCCCTCA GCCTCCCAGGTAGCTGGGACTACACCAC ACTGGGACTACACCAGCTGCCACCATGC CTAG
CTAATTTTTGTATTTTTGGTAGAGACAGG GTTTTGCCGTGTTGCCCAGGCTGGTAGAT CGG3'

The sequence of Ophiocomina nigra transcriptome, in 5'-3' shows the following nucleotides:

>TRINITY_DN49978_c0_g1_i1 (ICAM1)

5'GATTGATAAGTCATATGATATAAAATT TGATTTTTTTTTTTTTTCCTATTTTTTGTT GTT

GAAAATTTATACTTGTCAGTATTTGGGGTT TTCACTTGTGGAGACCAATAAAGTGTATCT

CCAGTTTTGTTTGGAAATCGTTCATTCAGT GTGTGATAACGCGATTACAGTACTTGTGGT

GCAATCAATTATAATGTGGATCAAACTT CTCAAATGTTTAATTGTGATGTGTGGCGT GTGCTTCAAATAAGCACTGAAT3'

\section{Conclusion}

Genes from cells showing the antigen (such as CXCR4 gene, PECAM1 gene and ICAM1 gene) are present in Invertebrates.

CXCR4 gene is, in human, in relation with immunodeficiences and cancer. We suppose it is in correlation with immune defence in Echinodermata. PECAM1 gene plays a rôle in the platelet'physiology. We recall we discovered, the IPA (Invertebrate Primitive Antibody) and the platelets(Ref : 1-2-3) in these same Echinodermata. Further studies are necessary to determine the exact rôle of ICAM1 gene, in these last ones. But we suppose it is in relation to Nuclear-Factor B we found also, in Invertebrates.

\section{REFERENCES}

[1] Leclerc, M (2020) IJRSMHS in press

[2] Leclerc, M. et al (2018) Int.J Vaccines Vaccin 5(1) : 00095

[3] Leclerc, M. et al (2018) Cell Cellular Life. Sci. J. 3 (1) 000117

[4] Grabherr, M.G et al (2011) Nature Biotechnology 29 644-652

[5] Altschul, S.F et al (1990) J.Mol.Biol 215(3) 403-410

Citation: Michel Leclerc, "Evidence of Cxcr4 Gene, Pecaml Gene, Icam1 Gene, from Cells Showing the Antigen, in Invertebrates", International Journal of Research Studies in Medical and Health Sciences. 2020; 5(4): 29-30.

Copyright: (c) 2020 Michel Leclerc, This is an open-access article distributed under the terms of the Creative Commons Attribution License, which permits unrestricted use, distribution, and reproduction in any medium, provided the original author and source are credited. 